\title{
BMJ Open The burden of non-specific chronic low back pain among adults in KwaZulu- Natal, South Africa: a protocol for a mixed-methods study
}

\author{
Morris Kahere (D) , ${ }^{1}$ Themba Ginindza ${ }^{2}$
}

To cite: Kahere M, Ginindza T. The burden of non-specific chronic low back pain among adults in KwaZulu-Natal, South Africa: a protocol for a mixedmethods study. BMJ Open 2020;10:e039554. doi:10.1136/ bmjopen-2020-039554

- Prepublication history for this paper is available online. To view these files, please visit the journal online (http://dx.doi. org/10.1136/bmjopen-2020039554).

Received 19 April 2020 Revised 24 July 2020 Accepted 28 July 2020
Check for updates

(c) Author(s) (or their employer(s)) 2020. Re-use permitted under CC BY-NC. No commercial re-use. See rights and permissions. Published by BMJ.

${ }^{1}$ Department of Public Health Medicine, College of Health Sciences, University of KwaZuluNatal, Durban, South Africa ${ }^{2}$ Discipline of Public Health, School of Nursing and Public Health, University of KwaZuluNatal, Durban, South Africa

Correspondence to

Dr Morris Kahere;

mrrskhr@gmail.com

\section{ABSTRACT}

Introduction Low back pain (LBP) is a major public health concern, affecting individuals of all age groups across the world. In about $90 \%$ of LBP cases, there is no specific cause identified and is, therefore, referred to as non-specific LBP. Due to the non-specific nature of LBP, investigations such as radiological and laboratory investigations are unnecessary and results to delayed diagnosis and improper treatment culminating in LBP progressing into chronic LBP (CLBP). LBP is now the leading cause of disability with a significant socioeconomic burden. Despite all these challenges, CLBP is regarded as a trivial condition in low-and-middle-income countries and remains poorly investigated. The distribution of CLBP in Africa is unclear.

Methods and analysis The research will be conducted in two phases. The initial phase will be an observational, cross-sectional hospital-based study that will be recruiting 650 participants, to determine the prevalence and risk factors of CLBP. A standardised questionnaire will be used to collect baseline data on the socio-demographic characteristics of participants and other variables of interest (exercise history, occupational posture, level of education and the income status). Disability will be assessed using the Oswestry Disability Questionnaire and the psychological risk factors will be assessed using the IIIness-Behaviour Questionnaire (IBQ) and the FearAvoidance Belief Questionnaire (FABQ). The second phase will be a retrospective, top-down, prevalence-based cost-of-illness study of the 2018-2019 health records, to estimate the burden of CLBP from the healthcare system's perspective. The SPSS V.25.0 statistical package will be used for data entry and analysis. Statistical analysis will include descriptive statistics by means of graphs and cross tabulations, inferential statistics by means of logistic regression and $\chi^{2}$ test. A $p$ value of 0.05 will be deemed statistically significant.

Ethics and dissemination This protocol was approved by the University of KwaZulu-Natal's Biomedical Research Ethics Committee (Ref. No.: BREC/00000205/2019) and the KwaZulu-Natal Department of Health Research Ethics (Ref. No.: KZ_201909_002). This will be the first LBP costof-illness study in the sub-Saharan Africa, and, therefore, it will close these knowledge gaps and present important evidence on the estimated burden of CLBP in this context. The results of this study will be presented to the Department of Health and to the respective stakeholders

\section{Strengths and limitations of the study}

- The study will give an insight of the burden of low back pain (LBP) in Africa, including South Africa.

- It is the only study conducted in South Africa that reports on the economic burden of non-specific chronic LBP.

- The weakness of the study is that it does not have a control group.

- The sample size may not be large enough for generalisation of results and drawing of conclusions.

and decision-makers to discuss the findings and draw their attention to the prioritisation of LBP research, its management, prevention programmes and implementation of educational programme and for the planning of costcontainment policies.

\section{INTRODUCTION}

Low back pain (LBP) is the most prevalent musculoskeletal condition, increasingly becoming a major public health concern associated with significant disability and high economic costs in high-income countries. ${ }^{1}$ Years lived with disability caused by LBP has increased by $17.2 \%$ from the year 2005 to year 2015, accounting for a collective of 815 years lived with disability per 100000 persons globally. ${ }^{2}$ Activity-limiting LBP had a global point prevalence of $7.3 \%$ in 2015, which means that approximately 540 million people were affected at any given time. ${ }^{3}$ Disability associated with LBP differs significantly among nations due to different social norms and local healthcare approaches and legislation. ${ }^{3}$ A systematic review by Meucci et al, which included cross-sectional population-based studies and cohort studies on the prevalence of chronic LBP (CLBP) among adults, found a prevalence rate of $4.2 \%$ among individuals aged between 24 years and 39 years and a $19.6 \%$ among the adults aged between 20 years and 59 years. ${ }^{4}$ Another cross-sectional 
study by Iizuka et al reported a prevalence rate of nonspecific CLBP (NSCLBP) of $15.4 \%$ and a rate of $9.3 \%$ for specific CLBP among adults. ${ }^{5}$ Among the Brazilian adult population, CLBP had a prevalence rate of $25.4 \% .{ }^{4} \mathrm{~A}$ cross-sectional study by Freburger et al found the prevalence of CLBP to be on the rise from $3.9 \%$ in 1992 to $10.2 \%$ in $2006 .^{6}$

In a systematic review of population-based studies among children/adolescents and adults conducted in Africa, ${ }^{7}$ the lifetime prevalence of LBP was found to be $47 \%$, the annual prevalence was estimated at $57 \%$ and the point prevalence was estimated at $39 \% .^{7}$ A hospital-based, cross-sectional study by Dlungwane et al investigated the prevalence of LBP among nurses in KwaZulu-Natal (KZN), South Africa, and found a point prevalence of $59 \%$ with bending, prolonged positions and transferring patients reported as the factors strongly associated with LBP. ${ }^{8}$ Docrat's population-based study in 1999 reported an LBP prevalence rate of $45 \%$ among the Indians and $32.6 \%$ among the Coloureds in KZN province with an increased number of children and/or pregnancies, a low-level educational status and driving for long distances as significant predictors of LBP. ${ }^{9}$ A cross-sectional study by Major-Helsloot (2014) on the management of LBP at primary care levels in South Africa reported a lifetime prevalence of LBP of $73.2 \%$ with $26.3 \%$ suffering from CLBP. $^{6}$ The majority of LBP studies in South Africa report on work-related LBP epidemiological estimates or involve a specific population group, for example, pregnant women ${ }^{47}$ or adolescents. ${ }^{4}$ Hence, the prevalence of LBP among the general adult population in South Africa is not known.

In addition to the global rising prevalence of CLBP and its associated socioeconomic burden, LBP is also associated with significant disabilities and comorbidities. ${ }^{10} 11$ CLBP has been shown to be strongly associated with a reduction in sexual activity. ${ }^{12}{ }^{13}$ Insomnia has also been frequently reported as a consequence of CLBP. ${ }^{14}$ Some studies report an association of CLBP with earlier death, as they hypothesise that CLBP normally results in significant disability, which will cause activity limitation due to fear-avoidance behaviour. ${ }^{15} 16$ Over time this will result in complications of other existing comorbid conditions such as hypertension, diabetes mellitus, hypercholesterolaemia and a variety of other systemic conditions, which get execrated by overweight due to lack of exercise. ${ }^{17}$

LBP is defined as "pain, muscle tension or stiffness localised below the costal margin and above the inferior gluteal folds, with or without leg pain (sciatica) and described as chronic if it persists more than 12 weeks'. LBP is classified as specific if there is a known cause such as hernia, vertebral fracture, Pott's disease, tumours, etc or non-specific if there is no identifiable cause. ${ }^{18}$ Only about $10 \%$ of LBP cases have a known cause; hence, specific and $90 \%$ of the cases fall under the non-specific LBP category. ${ }^{18} 19$ However, previous studies have used inconsistent definitions of LBP, preventing cross-study comparisons, or have used different definition over time, culminating in varying conclusions on trends in epidemiological estimates. ${ }^{20} 21$

The burden of LBP is multifaceted and includes but not limited to pain, disability, carer burden, financial burden and healthcare resource utilisation. ${ }^{22}{ }^{23}$ In the USA, the annual direct cost of LBP has been estimated to be US $\$ 100$ billion, and $€ 2$ billion to $€ 4$ billion in Europe per year. ${ }^{24}{ }^{25}$ Approximately half of the LBP cases in primary care fluctuating pain of low-to-moderate intensity, some recover and some progress to persistent disabling severe back pain. ${ }^{3}$ The majority of LBP cases resolve within 2-6 weeks and only a small percentage progresses to persistent/chronic disabling back pain. ${ }^{3}$ According to Geurts $e t a l$, the prevalence of CLBP is estimated at $9 \%$ and is responsible for over $60 \%$ of the gross national product in the Netherlands. ${ }^{26}$ Over $90 \%$ of the socioeconomic burden of LBP is attributed to this small percentage of CLBP cases. ${ }^{27}$ However, the direct cost of LBP has not been investigated in South Africa. Therefore, the proposed study seeks to investigate the prevalence, risk factors and the economic burden of CLBP among adults in KZN, South Africa.

\section{Study rationale}

The purpose of this study is to establish the burden of CLBP among adults in KZN using a prevalence-based model. Knowledge of disease burden is important for decision-making in the public health sector. In order to establish appropriate policies, decision-makers need to know the magnitude of the disease in the population, the associated risk factor of and the trends of CLBP. ${ }^{14}$ The burden of CLBP is unknown in South Africa; therefore, the results of this study will add on to that knowledge gap and will provide vital information for policy strengthening or/and development of CLBP prevention and management, planning and research prioritisation in healthcare. A precise approximation of the health status of the population is needed to determine the expected healthcare resource utilisation and it is important for prioritising effective interventions and evaluating the impact of these interventions and their cost effectiveness. ${ }^{15}$ The local data on disease burden especially data on LBP is crucial to the government and other stakeholders for planning and establishing and/or strengthening prevention and management strategies. Based on the findings of this study, recommendations will be made on how to reduce the burden of CLBP at national, community and individual levels by targeting the risk factors that will be identified.

\section{Conceptual framework}

The pathophysiological mechanisms and the aetiological factors of NSCLBP have been unclear, but the biopsychosocial model seemed to have gained a wide acceptance for explaining the perpetuation of symptoms in CLBP. ${ }^{28}$ Symptomatic treatment without a definitive diagnosis is like treating blindfolded. The delay in addressing the root cause results in a cascade of events leading acute 
cases developing into chronic. The persistent perception of pain necessitate a fear-avoidance behaviour, which will catastrophise the illness, increasing rumination with a decrease in self-efficacy and an increase in the sense of helplessness. ${ }^{29}$ Angle's (2017) concept of illness suggests that the progression of pain stems from a stimulus, which leads to distress, illness behaviour and ultimately an adoption of a sick role, thus incorporating the biosocial, psychosocial and the social components into the model. ${ }^{30}$ A biomechanically research-based model of diagnosis, which incorporates the anatomical origins of pain, tissue load (compressive, tensile or shearing force) and identified faulty movement patterns, has been neglected in rehabilitation science. ${ }^{29}$ The postural theorem postulated by Mafuyayi et al describes the biomechanical causes of NSCLBP based on the increase in virtual weight that results from postural changes of the lumbar spine. ${ }^{31}$ According to Mafuyai et al (2014), people's weight varies with the changes in the posture of the lumbar spine which in turn varies the amount of stress that each anatomical structure of the lower back bears. The two postures of the lumbar spine are the lordotic and the kyphotic postures. In the lordotic posture, the virtual weight and stress on the lumbar spine are positive, increasing the weight and stress on the lower back. ${ }^{29}{ }^{31}$ In a kyphotic posture, virtual weight and stress are negative, reducing the original stress and pressure on the lower back. Nonspecific LBP is presumed to be caused by the lordotic posture which results in induced repetitive micro-injuries sustained by the structural parts of the lumbar spine as a result of increased weight and stress brought about by this posture. ${ }^{31}$ This type of posture is often seen on sedentary workers who spend most of their time sitting in front of a computer. However, the biomechanical explanation of NSCLBP in its entirety, has lost recognition to the holistic biopsychosocial model which is multifactorial in nature. ${ }^{32}$

\section{Aim}

The aim of the study is to establish the burden of CLBP among adults in KZN using the prevalence model.

\section{Specific objectives}

1. To determine the prevalence of CLBP among adults of 18 years and above presenting at selected public hospitals in KZN.

2. To identify risk factors associated with CLBP among adults of 18 years and above presenting at selected public hospitals in KZN.

3. To estimate the economic burden of CLBP using the prevalence model in KZN.

\section{Research questions}

1. What is the prevalence of CLBP among adults of 18 years and above presenting at selected public hospitals in $\mathrm{KZN}$ ?

2. What are the risk factors associated with CLBP among adults of 18 years and above presenting at selected public hospitals in KZN?
3. What are the costs associated with CLBP using the prevalence model in KZN?

\section{METHOD AND ANALYSIS \\ Study design}

The research will be conducted in two phases with two different designs. Phase one will be a cross-sectional hospital-based observational study, which will be conducted for the purpose of collection of baseline data on the prevalence, disabilities associated and risk factors (biomechanical and psychosocial) for CLBP. Phase two will be a retrospective, top-down, prevalence-based costof-illness (COI) study of the 2018-2019 health records (from five provincial hospitals in KZN, viz, Prince Mshiyeni Memorial Hospital, Mahatma Gandhi Memorial Hospital, Hillcrest Hospital, Addington Hospital and Clairwood Hospital) to estimate the direct economic cost associated with LBP from the healthcare systems' perspective. This will help to quantify the economic burden of LBP and also to illustrate the potential benefits of establishing a prevention programme.

\section{Ethical consideration}

This protocol was approved by the UKZN's Biomedical Research Ethics Committee (BREC) (Ref. No.: BREC/00000205/2019) and the KwaZulu-Natal Department of Health Research Ethics (Ref. No.: KZ_201909_002). Gatekeeper permission will be sought from participating institution prior to seeking signed informed consent from individual participants. Participation of all respondents in the study will be strictly voluntary. Measures will be taken to ensure the respect, dignity and freedom of each individual participating in the study. To guarantee the anonymity of each participant, the names of respondents, their addresses or other identifying information will not be included in the questionnaires. The study results will be presented to the Department of Health and to the respective stakeholders and decisionmakers to discuss the findings of the study and draw their attention to the prioritisation of LBP research, its management, prevention programmes and implementation of educational programme and for the planning of cost-containment policies. The results will also be presented in regional and international conferences and the findings published in peer-reviewed journals.

\section{Study area}

The study will be conducted in KZN because of its multiracial nature, which reduces the risk of selection bias and tend to give participants from all the different ethnic groups an equal chance to participate. KZN is a South African east coastal province with a population of approximately 11074800 people, which is about $19.6 \%$ of the total South African population and it covers the geographical area of $94361 \mathrm{~km}^{2}$. The province consists of about $19 \%$ of the youth aged $15-24$ years, $28 \%$ of the middle-aged adults (25-44 years), 13\% aged 45-64 years and only about $5 \%$ consists of the elderly aged over 65 
years, with a 49:51 male-to-female ratio. KZN borders three other South African provinces and the countries of Mozambique, Swaziland and Lesotho.

\section{Study settings}

This hospital-based study will be conducted on five randomly selected provincial primary public hospitals in KZN, viz, Addington Hospital (571 beds), Mahatma Gandhi Memorial Hospital (350 beds), Prince Mshiyeni Memorial Hospital (1200-bedded facility), Hillcrest Hospital (175 beds) and Clairwood Hospital (275-bed site). The KZN Department of Health estimated a total caseload of 28.4 million in the year 2019 .

\section{Study participants and recruitment}

The participants in this cross-sectional study will be adult men and women aged 18 years and above living in KZN. Several approaches will be used to enhance participation in this project. The study will be advertised in local newspapers and broadcasted in local radio stations in order to inform the general population and create awareness. Recruitment shall also occur via poster advertisement that will be placed on the walls of the selected public hospitals' outpatient department. Participants attending the participating hospitals during the study period will be approached by the research assistants, inviting them to participate. The research assistants will verbally explain the purpose and procedures of the research to participants. Those that are interested to participate will be given a letter of information to read and understand. At that stage, those that agree to participate will be given an informed consent to read and sign in their handwriting. The data collection procedure will only commence once the final review comments have been received and will proceed until the required sample size is reached. We anticipate that the research study data collection process will take up to 6 months from the day of commencement of data collection.

\section{Eligibility criteria}

Adult participants aged 18 years and above presenting at the selected public hospitals who are willing to sign the informed consent will be recruited into the study. Those that do not satisfy the criteria for inclusion will be excluded, including those that are mentally ill, physically disabled, those with congenital anomalies such as cerebral palsy, ambulatory issues and other serious medical conditions.

\section{Sampling and sample size}

First, a simple random sampling was done for the study sites selection. There are 18 provincial hospitals in KZN and the name of each hospital was written on a small piece of paper, all equal in size and shape. These pieces of paper were all put in a hat and the hat was shaken five times. An independent person who is not part of the research team picked the papers from the hat without seeing them, and the first five to be picked were selected as study sites. Second, the study will apply the systematic random sampling for participant selection purpose, where every third person (after the first one being recruited using the lottery method) presenting at the selected study site will be recruited until the required sample size is achieved.

The appropriate sample size for this study will be determined largely by three factors: (1) the estimated prevalence of the outcome of interest, (2) the desired level of confidence $(95 \%)$ and (3) the acceptable margin of error $(5 \%)$. The prevalence of CLBP in KZN is unknown; therefore, an assumed prevalence of $50 \%$ will be used to estimate the sample size of the study using the following formula:

$$
\mathrm{n}=\frac{\mathrm{z}^{2} \mathrm{p}(1-\mathrm{p})}{\mathrm{d}^{2}}
$$

where

$\mathrm{n}=$ required sample size.

$\mathrm{z}=$ confidence level at $95 \%$ (standard value of 1.96).

$\mathrm{p}=$ assumed prevalence of CLBP in KZN ( $50 \%)$.

$\mathrm{d}=$ margin of error at $5 \%$ (standard value of 0.05 ).

Based on the assumed prevalence of CLBP, the estimated sample size will be 384 . The sample will further be increased by a margin of $10 \%$ to account for contingencies such as that of non-response or recording error, and also be increased by design effect to correct for any difference in design. The sample size will be multiplied by the design effect (D), which is normally multiplied by 1.5. This is in accordance with the recommendations by Fearon $e t a l$, who states that the sample size of the simple random sampling must be multiplied by the design effect. ${ }^{33}$ Increasing the sample size will reduce the type I and type II errors and known and unknown confounders' effects. ${ }^{33}$ Therefore, the final sample size needed for the study is 650 adults.

\section{Study instruments and data collection}

A standardised structured questionnaire will be used to collect baseline data (detailed information concerning socio-demographic characteristics and other lifestyle characteristics, and clinical presentations data from participants). This standardised tool will also gather information on the biomechanical risk factors of LBP, which include questions on exercise (frequency, types of exercises or sports, number of sessions and the duration of sessions per week) and postural habits (sitting, standing and driving postures, and posture on carrying/ lifting objects). Questions on the social determinants of CLBP will include aspects of the level of education, occupational and financial status of the participants. The psychological risk factors of CLBP will be assessed using the known IBQ ${ }^{34} 35$ and the FABQ. ${ }^{36}$ Disability associated with CLBP will be assessed using the Oswestry Disability Questionnaire, which is a known tool that has been tested for its reliability and validity. ${ }^{3738}$

All questionnaires will be recorded and sent with a questionnaire $\log$ form to the research office. The questionnaires and consent forms will be developed (the content of the data collection tools will be informed by the literature) in English and then translated to the local 
language (isiZulu). The completed questionnaires will be thereafter re-translated into English. Such a translation will involve an independent translator who will have no connection with the research team and not working for the researcher. The questionnaires will be precoded and closed ended. They will be then pretested among participants seeking similar health services from two sites (King DinuZulu Hospital and King Edward VII's Hospital), which will not participate in the study. Modifications will be made where necessary after pretesting the questionnaire. Thereafter, the standardised questionnaire will be used during the 5-day data collection training with the research assistants.

\section{COI estimation}

A prevalence-based COI study will be used to investigate the economic burden of CLBP. We will be using a topdown approach for cost associated with hospital care and a bottom-up approach to estimate cost associated with outpatient and primary care. The proposed prevalencebased top-down regression will rely on aggregated data (2018-2019 health records) and a model will be developed to combine epidemiological and economic data on LBP within the study context. The cost data will be estimated as per cost per patient and based on a 1-year (12 months) prevalence estimate. The aggregated costs will be presented in dollars per annum. This COI study will be conducted from the provider perspective since the state bears the majority of the costs of screening and treatment in South Africa. All identifiable direct medical costs were considered as primary diagnoses during 2020. All costs will be computed at the price level of 2020 and converted to US\$. A mix of bottom-up micro-costing ingredients and top-down approaches will be used to collect data on costs. A costing assessment tool will be developed to retrieve the necessary data on the direct medical care cost associated with LBP. These will include all costs on consultations, diagnostic procedures (imaging studies, laboratory tests and other special investigations), treatment procedures, medication, hospitalisations, referrals to specialists (orthopaedic surgeons, spinal surgeons or neurologists), physical therapy, rehabilitation, overhead costs allocated to technology and variable cost of utilities.

\section{Data management}

Questionnaires signed informed consent forms and questionnaire $\log$ forms will first be stored in locked cabinets at the study sites. Thereafter, they will be similarly kept at the Department of Public Heath Medicine offices (UKZN), and copies of the results will be sent to and stored by the nurses at the different sites. The questionnaires and outcome forms will be linked with the study/patient identification number. At the office, the data will be double entered into EpiData V.3.02 for Windows (The Epi Data Association Odense, Denmark), and then transferred to STATA V.13.0SE (Stata Corp, College Station, Texas) for analysis. Access to the server will be limited to the investigators. Server backup procedures will be managed by the University's Information and Communication Services Division. No participant identifiers will be included in the database. The data will always remain confidential. The BREC and the investigator will have access to the data. The data will be kept for 5 years according to the ethical committees' requirements at the Discipline of Public Health Medicine, after which it shall be shredded, and all electronic files erased.

\section{Data quality assurance}

A data quality system will be put into place to ensure the capturing of quality data for analysis. Data collection will be standardised, and instructions for completing the questionnaires will be developed. Periodical reviews of the data collection will be undertaken by the investigator. Such measures will help to identify gaps in data collection and patient recruitment.

\section{Data analysis overview}

Three components of analysis will be applied to this study, namely, descriptive, inferential and costing analyses. Summary of the analysis are listed in table 1 .

1. Descriptive statistics using frequency and crosstabulation tables and different types of graphs (including pie charts and bar graphs).

2. Inferential statistics by means of Pearson's and/or Spearman's correlations will be used, $\chi^{2}$ tests for the testing of hypothesis for nominal and ordinal data and

Table 1 Different aspects of the study with their objectives

\begin{tabular}{|c|c|c|}
\hline Objective & Data outcomes & Data analyses \\
\hline $\begin{array}{l}\text { To determine the prevalence of chronic low } \\
\text { back pain among adults of } 18 \text { years and above } \\
\text { presenting at selected public hospitals in } \\
\text { KwaZulu-Natal }\end{array}$ & $\begin{array}{l}\text { Prevalence of chronic low back pain } \\
\text { ORs (unadjusted and adjusted) and } \\
95 \% \text { Cls for potential risk factors } \\
\text { associated with outcome }\end{array}$ & $\begin{array}{l}\text { Descriptive analysis } \\
\chi^{2} \text { test to test the hypothesis }\end{array}$ \\
\hline $\begin{array}{l}\text { To identify risk factors associated with chronic } \\
\text { low back pain among adults of } 18 \text { years and } \\
\text { above presenting at selected public hospitals } \\
\text { in KwaZulu-Natal }\end{array}$ & $\begin{array}{l}{ }^{*} \text { ORs (unadjusted and adjusted) and } \\
95 \% \text { Cls for potential risk factors } \\
\text { associated with low back pain }\end{array}$ & $\begin{array}{l}\text { Logistic regression model for the } \\
\text { critical analysis of the risk factors } \\
\text { of chronic low back pain }\end{array}$ \\
\hline $\begin{array}{l}\text { To estimate the economic burden of chronic } \\
\text { low back pain using the prevalence model in } \\
\text { KwaZulu-Natal }\end{array}$ & $\begin{array}{l}\text { Annual estimated costs associated } \\
\text { with screening/diagnosis, managing } \\
\text { and treating of low back pain }\end{array}$ & $\begin{array}{l}{ }^{*} \text { Cost analysis using: } \\
\quad{ }^{*} \text { Prevalence-based cost-of-illness } \\
\text { analysis (economic evaluation) }\end{array}$ \\
\hline
\end{tabular}


the logistic regression analysis for the critical analysis of risk factors.

3. Costing analysis to establish the economic burden of LBP using a prevalence-based COI approach.

The table above illustrates the statistical aspects of each study objective.

\section{DISCUSSION}

The proposed study seeks to determine the evidencebased knowledge on the current prevalence of CLBP in South Africa and the associated economic burden. This will be the first COI study in South Africa to investigate the economic burden of LBP. Therefore, this study will highlight important knowledge to the policy-makers and other respective stakeholders within the public health fraternity to ensure an efficient allocation of healthcare resources and research prioritisations. The literature on LBP has been on the rise in the last decade in the African context, which can be evidenced by several systematic reviews done within this context. ${ }^{73940}$ However, there is little CLBP evidence. Most studies in Africa have described CLBP secondarily as a subheading on studies investigating other musculoskeletal conditions and not as a primary outcome measure. Therefore, the proposed study seeks to pave the way into the prioritisation of CLBP research, particularly its associated risk factors for chronicity and the socioeconomic burden. Based on the findings of this study, recommendations will be made on how to reduce the burden of CLBP by targeting the identified misconceptions and risk factors.

\section{Patients and public involvement}

There is no involvement of patients at this stage of the protocol development.

\section{Risk, benefits and standard of care \\ Risk}

This study involves no harm or human risk. All patients will be required to read and sign the letter of information and the informed consent. The names of all participants, their addresses or other identifying information will not be recorded in the questionnaire to ensure anonymity of each participant.

\section{Potential benefit}

The results of this study will serve as a wake-up call to policy-makers and government stakeholders for them to consider and allocate sufficient resources (funding) to LBP research since it is associated with a significant economic burden. This project will also help researchers to consider doing cost-benefit analysis research rather than just the COI study to come up with strategic measure to reduce the economic and societal burden of illnesses. The participants will be educated on the factors that are associated with the development of LBP and its progression to CLBP to promote a good health seeking behaviour.

\section{Standard of care}

An equal standard of care will be provided to all participants. All patients with LBP will be advised of the best conservative treatment/management options available; nutritional and exercise advice will be given as well.

Acknowledgements The authors would like to thank the University of KwaZuluNatal (UKZN) for the provision of resources towards this review and the UKZN Systematic Review Unit for the training and technical support. Participation of all respondents in the study will be strictly voluntary. Measures will be taken to ensure the respect, dignity and freedom of each individual participating in the study. To guarantee the anonymity of each participant, the names of respondents, their addresses or other identifying information will not be included in the questionnaires.

Contributors MK conceptualised the study protocol under the supervision of TG and designed data collection methods. MK and TG contributed to writing the first draft of the manuscript. Both the authors critically reviewed and approved of the final manuscript.

Funding The authors have not declared a specific grant for this research from any funding agency in the public, commercial or not-for-profit sectors.

Competing interests None declared.

Patient and public involvement Patients and/or the public were not involved in the design, or conduct, or reporting, or dissemination plans of this research.

Patient consent for publication Not required.

Provenance and peer review Not commissioned; externally peer reviewed.

Open access This is an open access article distributed in accordance with the Creative Commons Attribution Non Commercial (CC BY-NC 4.0) license, which permits others to distribute, remix, adapt, build upon this work non-commercially, and license their derivative works on different terms, provided the original work is properly cited, appropriate credit is given, any changes made indicated, and the use is non-commercial. See: http://creativecommons.org/licenses/by-nc/4.0/.

ORCID iD

Morris Kahere http://orcid.org/0000-0003-3669-9768

\section{REFERENCES}

1 Cezarino RS, Cardoso JR, Rodrigues KN, et al. Chronic low back pain in patients with systemic lupus erythematosus: prevalence and predictors of back muscle strength and its correlation with disability. Rev Bras Reumatol Engl Ed 2017;57:438-44.

2 Doualla M, Aminde J, Aminde LN, et al. Factors influencing disability in patients with chronic low back pain attending a tertiary hospital in sub-Saharan Africa. BMC Musculoskelet Disord 2019;20:25.

3 Hartvigsen J, Hancock MJ, Kongsted A, et al. What low back pain is and why we need to pay attention. Lancet 2018;391:2356-67.

4 Meucci RD, Fassa AG, Faria NMX. Prevalence of chronic low back pain: systematic review. Rev. Saúde Pública 2015;49:73.

5 lizuka Y, lizuka H, Mieda T, et al. Prevalence of chronic nonspecific low back pain and its associated factors among middle-aged and elderly people: an analysis based on data from a musculoskeletal examination in Japan. Asian Spine J 2017;11:989-97.

6 Freburger JK, Holmes GM, Agans RP, et al. The rising prevalence of chronic low back pain. Arch Intern Med 2009;169:251-8.

7 Morris LD, Daniels KJ, Ganguli B, et al. An update on the prevalence of low back pain in Africa: a systematic review and meta-analyses. BMC Musculoskelet Disord 2018;19:196.

8 Dlungwane T, Voce A, Knight S. Prevalence and factors associated with low back pain among nurses at a regional hospital in KwaZuluNatal, South Africa. Health SA Gesondheid 2018;23.

9 Docrat A. A comparison of the epidemiology of low back pain in Indian and coloured communities in South Africa, 1999.

10 Global Burden of Disease Study 2013 Collaborators. Global, regional, and national incidence, prevalence, and years lived with disability for 301 acute and chronic diseases and injuries in 188 countries, 19902013: a systematic analysis for the global burden of disease study 2013. Lancet 2015;386:743-800.

11 Igwesi-Chidobe CN, Coker B, Onwasigwe CN, et al. Biopsychosocial factors associated with chronic low back pain disability in rural Nigeria: a population-based cross-sectional study. BMJ Glob Health 2017;2:e000284. 
12 Grabovac I, Dorner TE. Association between low back pain and various everyday performances. Wien Klin Wochenschr 2019;131:541-9.

13 Odole AC, Olugbenga-Alfred AA. Sexual functioning and selected clinical and psychosocial factors among individuals with chronic non-specific low back pain in Ibadan, Nigeria. Sex Disabil 2018;36:185-94.

14 Majid Bet al. Frequency and severity of insomnia in chronic low back pain. Rawal Medical Journal 2017;42:528-30.

15 Martin CJ, Jin C, Bertke SJ, et al. Increased overall and causespecific mortality associated with disability among workers' compensation claimants with low back injuries. Am J Ind Med 2020;63:209-17.

16 Fernandez M, Boyle E, Hartvigsen J, et al. Is this back pain killing me? all-cause and cardiovascular-specific mortality in older Danish twins with spinal pain. Eur J Pain 2017;21:938-48.

17 Smith D, Wilkie R, Croft P, et al. Pain and mortality: mechanisms for a relationship. Pain 2018;159:1112-8.

18 Maher C, Underwood M, Buchbinder R. Non-Specific low back pain. The Lancet 2017;389:736-47.

19 van Middelkoop M, Rubinstein SM, Verhagen AP, et al. Exercise therapy for chronic nonspecific low-back pain. Best Pract Res Clin Rheumatol 2010;24:193-204.

20 Harkness EF, Macfarlane GJ, Silman AJ, et al. Is musculoskeletal pain more common now than 40 years ago?: two population-based cross-sectional studies. Rheumatology 2005;44:890-5.

21 Deyo RA, Mirza SK, Martin BI. Back pain prevalence and visit rates: estimates from U.S. national surveys, 2002. Spine 2006;31:2724-7.

22 Hoy D, Bain C, Williams G, et al. A systematic review of the global prevalence of low back pain. Arthritis Rheum 2012;64:2028-37.

23 Hoy D, Brooks P, Blyth F, et al. The epidemiology of low back pain. Best Pract Res Clin Rheumatol 2010;24:769-81.

24 Indrakanti SS, Weber MH, Takemoto SK, et al. Value-Based care in the management of spinal disorders: a systematic review of costutility analysis. Clin Orthop Relat Res 2012;470:1106-23.

25 Furtado RNV, Ribeiro LH, Abdo BdeA, et al. [Nonspecific low back pain in young adults: associated risk factors]. Rev Bras Reumatol 2014;54:371-7.

26 Geurts JW, Willems PC, Kallewaard J-W, et al. The impact of chronic discogenic low back pain: costs and patients' burden. Pain Res Manag 2018;2018:1-8.
27 Marin TJ, Van Eerd D, Irvin E, et al. Multidisciplinary biopsychosocial rehabilitation for subacute low back pain. Cochrane Database Syst Rev 2017;6:CD002193.

28 Farrokhi S, Mazzone B, Schneider M, et al. Biopsychosocial risk factors associated with chronic low back pain after lower limb amputation. Med Hypotheses 2017;108:1-9.

29 Asavasopon S. Chronification of low back pain: getting to the spine of the problem. Braz J Phys Ther 2018;22:1-6.

30 Bevers K, Watts L, Kishino ND, et al. The biopsychosocial model of the assessment, prevention, and treatment of chronic pain. US Neurol 2016;12:98-104

31 Mafuyai Met al. Postural theory of non-specific low back pain (NSLBP), 2013.

32 Shojaei I. Lower back biomechanics at Non-chronic stage of low back pain, 2018

33 Fearon E, Chabata ST, Thompson JA, et al. Sample size calculations for population size estimation studies using multiplier methods with respondent-driven sampling surveys. JMIR Public Health Surveill 2017;3:e59.

34 Aasdahl L, Marchand GH, Gismervik Sigmund Østgård, et al. The fear avoidance beliefs questionnaire (FABQ) does it really measure fear beliefs? Spine 2020;45:134-40.

35 Bid DDet al. Cross cultural adaptation, reliability and validity of Gujarati version of fear avoidance belief questionnaire in chronic low back pain. Natl J Integr Res Med 2016;7:1-8.

36 Rostami M, Noorian N, Mansournia MA, et al. Validation of the Persian version of the fear avoidance belief questionnaire in patients with low back pain. J Back Musculoskelet Rehabil 2014;27:213-21.

37 Chiarotto A, Maxwell LJ, Terwee CB, et al. Roland-Morris Disability Questionnaire and Oswestry Disability Index: which has better measurement properties for measuring physical functioning in nonspecific low back pain? Systematic review and meta-analysis. Phys Ther 2016;96:1620-37.

38 Lee C-P, Fu T-S, Liu C-Y, et al. Psychometric evaluation of the Oswestry disability index in patients with chronic low back pain: factor and Mokken analyses. Health Qual Life Outcomes 2017:15:1-7.

39 Morris L, Daniels K, Louw Q. The prevalence of low back pain in Africa. Man Ther 2016;25:e122-3.

40 Louw QA, Morris LD, Grimmer-Somers K. The prevalence of low back pain in Africa: a systematic review. BMC Musculoskelet Disord 2007;8:105. 